\title{
Untangling complexity: assessing the joint effects of population composition and context on perceived exposure to onchocerciasis in coastal Tanzania
}

\author{
Frederick Ato Armah ${ }^{1,2^{*}}$, Sheila A. Boamah ${ }^{3}$ \\ ${ }^{1}$ Department of Environmental Science, University of Cape Coast, Cape Coast, Ghana \\ farmah@ucc.edu.gh \\ ${ }^{2}$ Environmental Health and Hazards Lab, Department of Geography, University of Western \\ Ontario, London, ON, Canada, farmah@uwo.ca \\ ${ }^{3}$ School of Nursing, McMaster University, Hamilton, Canada, boamahs@mcmaster.ca \\ *Author to whom correspondence should be addressed
}

\begin{abstract}
Onchocerciasis volvulus is the second highest infectious cause of blindness in the world, and is estimated to affect 37 million people, of whom 99\% reside in sub-Saharan Africa. As a public health problem the disease is most closely associated with Africa, where it constitutes a serious obstacle to socio-economic development. Using the human ecology triad, this paper evaluates the dynamic interplay of population, habitat and behavioural factors in predicting perceived exposure to onchocerciasis among coastal inhabitants in Tanzania. Generalized linear models with log-log link function were fitted to cross-sectional survey data on 1253 individuals in three contiguous coastal regions. A significant proportion of respondents $(28 \%)$ perceived that they were exposed to onchocerciasis. Residents in urban locations irrespective of wealth status were less likely to report living in onchocerciasis endemic environment compared with their rural counterparts. This is understandable given that urban areas of Tanga and Dar es Salaam are definitely non-endemic and perceived risk of onchocerciasis is related to the fact of living in an endemic area with active onchocercasis transmission. Individuals who had attained secondary $(\mathrm{OR}=0.51, \mathrm{p}<0.01)$ or tertiary education $(\mathrm{OR}=0.37, \mathrm{p}<0.001)$, and reported easy access to health facility $(\mathrm{OR}=0.53, \mathrm{p}<0.001)$ were all less likely to report perceived exposure to onchocerciasis. This is not surprising because higher level of education and easy access to health facilities are characteristics of urban compared with rural areas. Policy implications suggest the need for the Tanzanian national neglected tropical disease control programme (TZNTDCP) to intensify health and educational campaigns at the community level and address susceptibility of vulnerable populations to the disease especially, for rural dwellers.
\end{abstract}

Keywords: onchocerciasis, perception, socio-environmental determinants, human ecology, coast, Tanzania 


\section{Introduction}

Onchocerciasis is caused by Onchocerca volvulus, a parasitic worm that lives for up to 14 years in the human body. It is spread through the bite of a blackfly of the Simulium damnosum species complex, which breeds in fast-flowing rivers and streams (Coffeng et al., 2013; Mackenzie et al., 2012; Taylor et al., 2014). It is the world's second leading infectious cause of blindness. According to Hotez et al. (2007), the prevalence of onchocerciasis, at the global level, is 37 million, and the population at risk is 90 million. Of those who are at risk of onchocerciasis worldwide, 99\% are in sub-Saharan Africa (Remme et al., 2006). Onchocerciasis is endemic in thirty African countries including Angola, Benin, Burkina Faso, Burundi, Cameroon, Central African Republic, Chad, Congo, Côte d'Ivoire, Democratic Republic of the Congo, Equatorial Guinea, Ethiopia, Gabon, Ghana, Guinea, Guinea-Bissau, Kenya, Liberia, Malawi, Mali, Mozambique, Niger, Nigeria, Rwanda, Senegal, Sierra Leone, Sudan, Togo, Uganda and the United Republic of Tanzania. The uneven spatial distribution of the disease is an important indicator of the nature and scope of endemicity, the local potential for transmission, as well as a predictor of the intensity and duration of interventions needed to control or eliminate it (Murdoch et al., 2002; WHO, 2010; Zoure et al., 2014).

Empirical evidence suggests significant strides in improvement of population health since the African Programme on Onchocerciasis Control (APOC) was established in 1995 (Amazigo, 2008), and almost 70 million people have been treated with ivermectin in 2009, reaching $75 \%$ of the target population of 90 million (Coffeng et al., 2013; Zoure et al., 2014). CommunityDirected Treatment with Ivermectin (CDTI) projects are currently operating in $96 \%$ of target countries protecting 94 million people most of who are in remote areas (Coffeng et al., 2013). There are 146,000 communities engaged in CDTI and also, a cumulative total of 1.3 billion ivermectin tablets have so far been delivered by communities (Zoure et al., 2014). It is estimated that 36 million people would have been affected in the APOC countries by 2011 if there had been no ivermectin treatment (Zoure et al., 2014).

Tanzania is one of the countries in sub-Saharan Africa where concerted efforts have been devoted to addressing onchocerciasis prevalence and treatment. Since 2009, all control activities are implemented through the integrated Tanzania National Neglected Tropical Diseases Control Programme (TZNTDCP). Currently, ivermectin is distributed in combination with albendazole as one drug package for lymphatic filariasis and onchocerciasis where there is overlap. To date, all meso and hyper endemic communities in affected districts are implementing mass drug administration (MDA) (i.e., 100\% geographic coverage) (see Noma et al. 2014; Zoure et al. 2014). The geographic distribution of onchocerciasis in Tanzania is well known (the mapping of onchocerciasis has been completed in Tanzania and the results published) (see Noma et al. 2014; Zoure et al. 2014). In the Tanga and Pwani regions in Tanzania, onchocerciasis is quite prevalent especially in the Usambara Mountains in Muheza district and a narrow strip at the western border of Bagamoyo district. However, in the rest of the study districts, the urban areas of Tanga and Dar es Salaam, onchocerciasis is not endemic as there are fewer transmission of Simulium vectors.

Notwithstanding the fact that the mapping of onchocerciasis has been completed in Tanzania, what remains unknown is people's perception of exposure to the disease and how population composition and contextual factors influence this perception. In contrast with many neglected 
tropical diseases (NTDs), the consequence of exposure to risk in onchocerciasis may give origin to clinically expressive infection or visual impairments many years or decades later. As a consequence, symptom recognition and perceived disease seriousness is usually very difficult to identify by trained people in the management of disease. Most of communities from different ethnic groups in African recognize the benefit of onchocerciasis treatment because of its additional action on intestinal parasites. Recognition of onchocerciasis by the community and the perceived risk of infection is strongly related to the level of endemicity.

Perceptions (i.e., beliefs about potential harm or exposure to diseases) are components of most theories of health behavior (Brewer et al., 2007; Ferrer \& Klein, 2015; Granado et al., 2011), and it has been shown to influence likelihood of behavioural change. Individual perceptions and attitudes are crucial inputs into healthy behaviours as they influence the pathways of symptom recognition, perceived disease seriousness, utilization of services, and eventual health outcomes (Granado et al., 2011). Given that there is a complex interplay between risk perceptions and health behaviour, it is important to understand how socio-environmental determinants influence perceived exposure to onchocerciasis. Uunderstanding the role of specific perceptions in motivating people to engage in precautionary behaviour may help health communicators to improve their messages about prevalence and transmission of onchocerciasis. In this study, we demonstrate the intricate relationship between compositional and contextual factors in influencing people's perceptions of onchocerciasis exposure in coastal Tanzania. Although the socio-environmental determinants of onchocerciasis are well-documented, we are still in the early stages of appreciating the full extent of the impacts that follow when the contextual factors that give rise to heterogeneities in perception of exposure to onchocerciasis are superimposed on the compositional factors. Taking into consideration the fact that onchocerciasis show a focal and heterogeneous geographical distribution due to a complex set of interactions between geological, ecological, entomological, social, anthropological and behavioral factors, it is imperative to understand relevant environmental (biophysical and social), behavioural and predisposing factors and to recognize the inherent complexity of composition/context/ collective effects that give rise to the disease and its associated perceptions. This is a fundamental objective of this study. Using a human disease ecology perspective, we draw attention to how population, habitat and behavioural factors dynamically interact to shape the perception of the disease.

We attempt to explain the perception of onchocerciasis prevalence within the framework of human disease ecology. The human disease ecology framework is one widely used theoretical model developed to study health behaviors but it has not been used in research on public perception of disease endemicity and exposure. A disease ecology perspective is one that situates human health within the context of a human being's interactions within a wide array of environments, including the physical environment that surrounds us as well as the social and cultural environments all humans are influenced by and participate in (Curtis and Oven, 2012; Meade, 2010; Smith et al., 2007). Meade and Earickson (2000) have consolidated the disease ecology concept by modeling the collection of environments humans are exposed to as the "triangle of human ecology" (see Figure 1). In this model, habitat, population, and behaviour each occupy one of the three vertices of a triangle, with a human being's state of heath, in this context, exposure to onchocerciasis, located somewhere within the enclosed space. Meade and 
Erickson (2000) consider these three groups as broad, elastic characterizations of the various aspects of human life. The modified human ecology triangle is the proposed framework used in this study linking socio-environmental determinants (population, behavioural and habitat factors) to perception of onchocerciasis exposure.

In the human ecology triangle, the population, the first vertex is concerned primarily with the genetic influences that are passed on from generation to generation within a given population. Factors such as sex, age, and ethnicity can be incorporated in this vertex. In the modified human ecology triangle, under population vertex, genetics has been substituted with ethnicity given that in this study the focus is not on biological susceptibility but rather on other characteristics of the population that may predispose individuals to the disease. Unlike the other two categories of disease ecology, humans are not able to exert any control over the factors considered under the population category (Meade, 2010; Meade and Emch, 2010). This is for the simple reason that each of us is born with a certain set of genes that, aside from possible gene therapy interventions, we cannot change. With these genes come predispositions to developing certain diseases and resistances to others. The second vertex on the human ecology triangle is behaviour. This is one of the important features of the disease ecology perspective because human beings are seen not as passively relating with their environment but rather as actively exerting influence over their natural, built, and social environments. The human trait of cultural expression is also included in this vertex (Meade, 2010; Meade and Emch, 2010). From culture, belief systems, values, and perceptions arise - all of which help to shape the way in which human beings choose to interact with their environment (Curtis and Oven, 2012). The third vertex on the human ecology triangle is habitat. Three distinct habitat spaces fall under this heading: the natural environment, which consists of surrounding topography, water, plants, animals, and climate conditions; the built environment, which consists of the urban and residential landscapes in which people live and work; and finally the social environment, which consists of the groups, relations, and societies within which people live (Curtis and Oven, 2012; Meade, 2010; Plowright et al., 2008; Smith et al., 2007). All humans are constantly interacting with each of these habitats, each to varying degrees. All three aspects - habitat, population, and behaviour -interact together and influence how people perceive their exposure to a particular disease. 


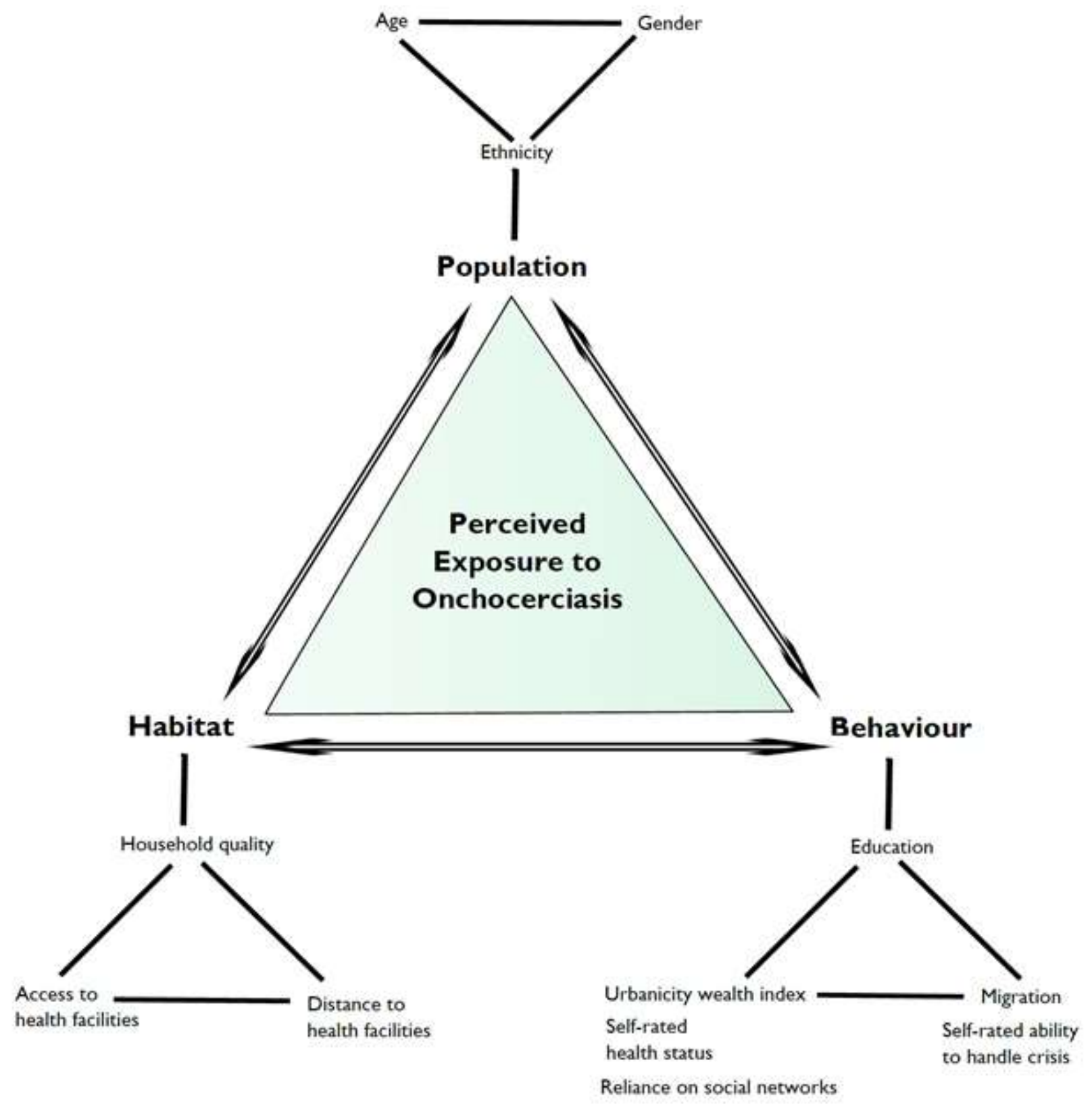

Figure 1: The human ecology triangle in relation to perceived onchocerciasis (modified from Meade and Earickson, 2005)

The consequences of onchocerciasis experienced by individuals living proximal to one another are more similar than to those living at a distance. In theory, three plausible explanations account for this observation. First, it may purely be that individuals in the same neighbourhood tend to have the same characteristics than to those in other neighbourhoods in terms of predisposing or biological factors (Pickett and Pearl, 2001) such as age, gender and ethnicity, that is, the composition effect. Another viable explanation may be related to geographical factors. Individuals living in the same neighbourhood are exposed to similar local factors (Pickett and Pearl, 2001) that have impacts on their exposure to onchocerciasis, for example, 
proximity to a river where blackfly proliferate or service provision, that is, the context effect. It can also be argued that individuals who live in proximity are more likely to engage in the same types of behaviour that may have influences on health outcomes - for example behaviour of bathing in rivers among adolescents may be affected by peer pressure-that is, the collective effect. Since we live in a complex world, in reality all three elements may be present to varying extents in relation to the distribution of onchocerciasis.

\section{Materials and Method}

\section{Study area}

Tanzania was chosen for three reasons. First, rapid epidemiological mapping shows that several communities are endemic and in need of community-directed treatment of ivermectin. Secondly, according to the African Programme on Onchocerciasis Control (APOC), the number of people within meso- and hyper-endemic onchocerciasis areas in Tanzania is $2,111,847$. This constitutes about $4.7 \%$ of the total population. Thirdly, there is paucity of research in Tanzania on how population, habitat and behavioural factors dynamically interact to shape public perceptions of onchocerciasis.

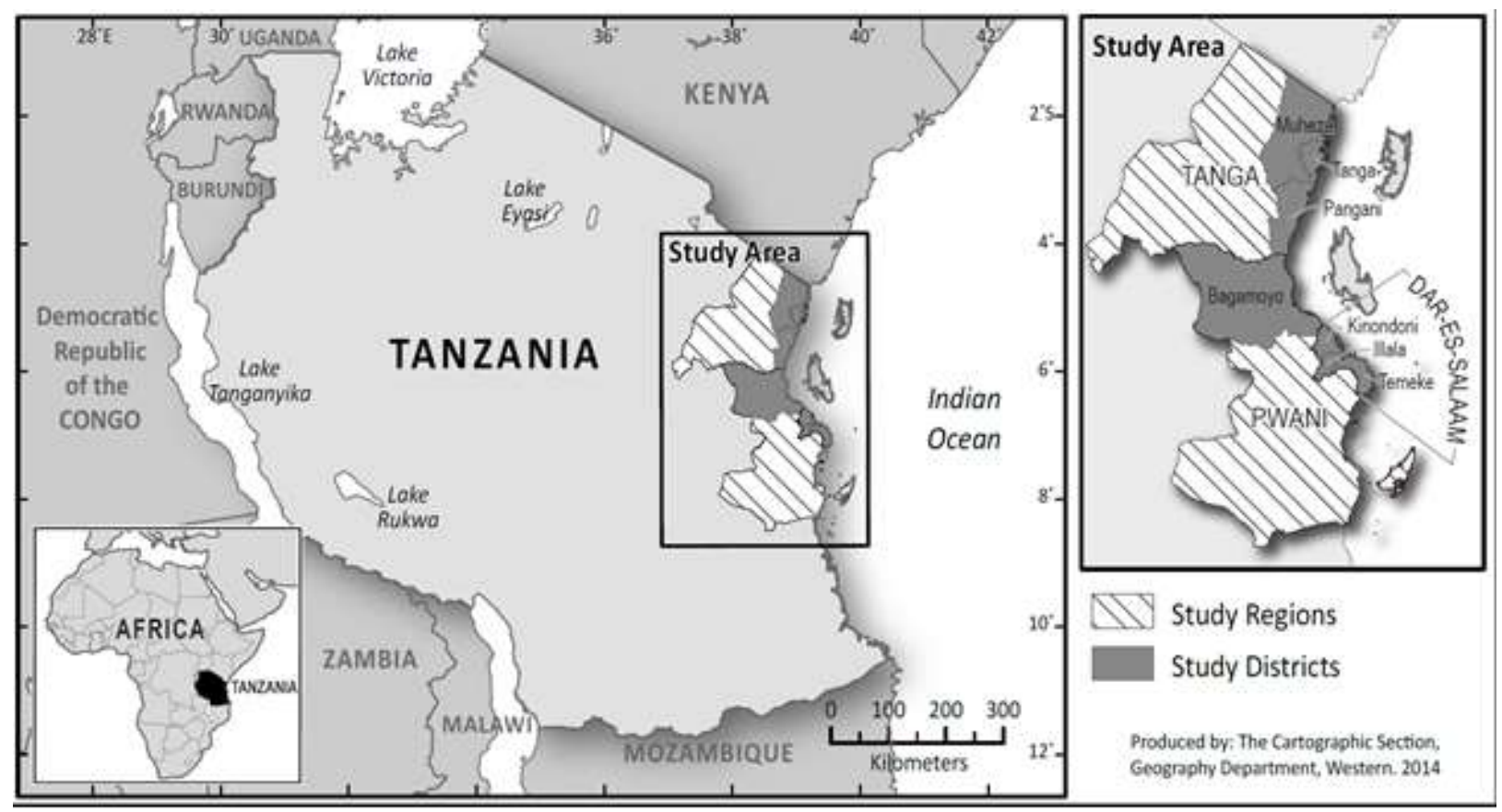

Figure 2: Map of Tanzania showing the study area

As shown in Figure 2, Tanzania is a coastal country lying between longitude $29^{\circ}$ and $49^{\circ}$ East and latitude $1^{\circ}$ and $12^{\circ}$ south of the Equator (Francis and Bryceson, 2001). The marine waters comprise $64000 \mathrm{~km}^{2}$ as territorial waters and $223000 \mathrm{~km}^{2}$ as offshore waters (EEZ) (Mngulwi, 2003). Tanzania's coastline stretches for $800 \mathrm{~km}$ covering about 15 percent of the country's total 
land area (TCMP, 2003). It has five coastal regions-Tanga, Pwani, Dar-es-Salaam, Lindi and Mtwara. However, this study specifically focused on Dar-es-Salaam, Pwani and Tanga regions. The population of Tanzania was estimated at 43 million in 2010 (National Bureau of Statistics, 2011).

\section{Data collection}

A cross sectional survey with 1253 individuals in three regions (Dar es Salaam, Tanga, and Pwani) along the coastline of Tanzania. The survey data were collected between March and September 2013. The study population included male (606) and female (647) participants between the ages of 18 and 70+ years. The study used multistage sampling to obtain representative estimates of the population of residents of the three regions. Within each region, a list of villages based on the 2012 Population and Housing Census was divided further into households. The list of villages was also divided into clusters ensuring that each cluster would provide adequate numbers of eligible respondents to be included in the survey. This approach both corrects for sampling bias and weights the cases to match census percentages of males and females of various age groups and by ethnicity (Armah et al., 2015a; b; Boamah et al., 2017). The enumeration areas (EAs) and their total number of households were listed geographically by urban and rural areas and EAs did not include the minimum number of households, geographically adjacent EAs were amalgamated to yield sufficient households (see Armah et al., 2015b). This provided the frame for selecting the clusters to be included in the survey according to a stratified systematic sampling technique in which the probability for the selection of any cluster was proportional to its size. A sampling interval was calculated by dividing the total number households by the number of clusters. A random number between 1 and the sampling interval was computer generated. The EA in which the random number fell was identified as the first selected cluster. The sampling interval was applied to that number and then progressively until the 20 (urban) and 15 (rural) clusters were identified. These clusters made up the sample for the survey. Households were randomly selected from these clusters for interview. Ethical approval for the study in was obtained from the Western University, Canada Non-medical Research Ethics Board. In Tanzania, study approval was granted by the Commission on Science and Technology (COSTECH).

\section{Measures}

Outcome variable

In urban areas of Tanzania, there is no onchocerciasis transmission, and therefore no exposure to onchocerciasis. However, some people still think they are exposed to the disease likely due to mediating factors such as previous experience, migration as well as compositional and contextual factors. The outcome of interest - perceived exposure to onchocerciasis - refers to what people think rather than actual clinical exposure to the disease. Each of the 1253 individuals in the survey were coded ' 0 ', that is, for those who indicated they were not exposed to the disease or coded ' 1 ', that is, for those who perceive they were exposed to the disease. On this basis, $28 \%$ of respondents (352 people) were considered to be exposed. This number is composed of 162 males and 190 females, respectively. 
Independent factors

Based on the human disease ecology framework, theoretically relevant variables were categorized into behavioural, population, and habitat related factors. Behavioural factors included urbanicity wealth index, educational attainment, self-rated health status, self-rated ability to handle personal crisis and difficulties, reliance on social network and migration (Figure 1). Recognizing that the nexus between health, poverty and urbanicity is rather complex, we generated a combined variable, which accounts for heterogeneities in social status within urban and rural areas, that is, urbanicity wealth index. This variable consists of six mutually exclusive groups: rural poor, rural middle, rural rich, urban poor, urban middle, and urban rich that were coded 1 through 6, respectively. Educational attainment was coded as none ' 0 ', primary education ' 1 ', secondary education ' 2 ' and tertiary education ' 3 '. Both self-rated health status, and self-rated ability to handle personal crisis and difficulties were coded was poor ' 0 ' and good ' 1 '. Reliance on social network was operationalized as a dummy variable. Regarding migration, movement within coastal areas was coded as 0 whereas movement from inland to coastal areas was coded as 1 .

Population factors included age, gender and ethnicity. Age comprises of four groups namely 18 $35,36-50,51-65$ and greater than 65 years; these were coded 1 through 4, respectively. Gender was operationalized as a dummy variable, with male as reference category. Ethnicity consists of three groups namely Zaramo, Sambaa and others, which were coded as 1, 2 and 3, respectively.

Habitat factors included relative household quality, access to nearest health facility and distance to nearest health facility. Household quality takes into account the quality of the built environment in relation to others within the same neighbourhood. This variable was operationalized as a dummy variable with poor as the reference category. Regarding access to nearest health facility, not easy and easy were coded as 0 and 1, respectively. The only covariate, distance to health facility, is an objective measure of how far respondents lived away from the nearest health facility. This variable consists of the measured distances in kilometers.

\section{Statistical analyses}

In this paper, four levels of analysis were employed. At the univariate level, the frequencies and percentage of sub-groups in the sample were presented to show the distribution of respondents in coastal Tanzania. At the bivariate level, Pearson's Chi-square test was conducted to examine significant relationships between independent variables (socio-environmental determinants) and exposure to onchocerciasis. At the multivariate level, two models were employed to examine the relationship between socio-environmental determinants and exposure to onchocerciasis; the first was an unadjusted multivariate model and the second model examined the relationship between the independent variables while adjusting for the effect of theoretically relevant variables.

The outcome variable used for analyses is dichotomous, but the cases are unevenly distributed. Approximately $28 \%$ of respondents reported perceived exposure whereas $72 \%$ reported no perceived exposure to onchocerciasis, implying that using a probit or logit link function that 
assumes a symmetrical (50\% - 50\%) distribution could produce biased parameter estimates. Thus, a log-log model that is suited for asymmetrical distributions, in which lower categories are more probable, was employed. However, the standard log-log models are built on the assumption of independence of observations but the cross sectional survey data has a hierarchical structure with participants nested within households and survey clusters. This means the response of participants in the same households and clusters may not be independent of each other, the result of which could potentially bias the standard errors. STATA 13, which provides an outlet for handling this problematic issue, is used by imposing on our models a 'cluster' variable, in this case the identification numbers of respondents at the cluster level. This in turn adjusts the standard errors producing statistically robust parameter estimates (Armah et al., 2015b).

\section{Results}

Sample Characteristics of Respondents

The demographic profile of the respondents is presented in Table 1. In coastal Tanzania, a significant proportion of respondents $(28 \%)$ reported perceived exposure to onchocerciasis. Approximately $33 \%$ and $24 \%$ of individuals resided in poor households in rural and urban areas, respectively. More than half of the respondents $(57 \%)$ had migrated from inland to coastal areas, and about $81 \%$ reported poor housing quality. Nearly half of the sample (46\%) had attained primary education. Majority of the participants $(84 \%)$ rated their health status as fair or worse and $67 \%$ rated their ability to handle personal crisis and unexpected difficulties as poor.

Table 1: Sample characteristics of respondents in coastal Tanzania $(n=1253)$

\begin{tabular}{lcr}
\hline & Frequency & $\%$ \\
\hline Oncho endemicity & & \\
Risk/ Exposure to onchocerciasis & & \\
No & 901 & 71.9 \\
Yes & 352 & 28.1 \\
Urbanicity wealth index & & \\
Rural poor & 417 & 33.3 \\
Rural middle & 75 & 6.0 \\
Rural rich & 18 & 1.4 \\
Urban poor & 297 & 23.7 \\
Urban middle & 225 & 18 \\
Urban rich & 221 & 17.6 \\
Education & & \\
No Education & 107 & 8.5 \\
Primary & 581 & 46.4 \\
Secondary & 343 & 27.4 \\
Tertiary & 222 & 17.7 \\
Self-rated health status & &
\end{tabular}




\begin{tabular}{|c|c|c|}
\hline Poor & 191 & 15.2 \\
\hline Fair & 858 & 68.5 \\
\hline Good & 204 & 16.3 \\
\hline \multicolumn{3}{|c|}{ Self-rated ability to handle personal crisis } \\
\hline Poor & 797 & 63.6 \\
\hline Good & 456 & 36.4 \\
\hline \multicolumn{3}{|c|}{ Self-rated ability to handle work crisis } \\
\hline Poor & 501 & 40.0 \\
\hline Good & 752 & 60.0 \\
\hline \multicolumn{3}{|l|}{ Reliance on social network } \\
\hline No & 1087 & 86.8 \\
\hline Yes & 166 & 13.2 \\
\hline \multicolumn{3}{|l|}{ Migration } \\
\hline No & 536 & 42.8 \\
\hline Yes & 717 & 57.2 \\
\hline \multicolumn{3}{|l|}{ Age } \\
\hline $18-35$ & 443 & 35.4 \\
\hline $36-50$ & 420 & 33.5 \\
\hline $51-65$ & 307 & 24.5 \\
\hline$>65$ & 83 & 6.6 \\
\hline \multicolumn{3}{|l|}{ Gender } \\
\hline Male & 606 & 48.4 \\
\hline Female & 647 & 51.6 \\
\hline \multicolumn{3}{|l|}{ Ethnicity } \\
\hline Zaramo & 245 & 19.6 \\
\hline Sambaa & 131 & 10.5 \\
\hline Others & 877 & 70 \\
\hline \multicolumn{3}{|l|}{ Household quality } \\
\hline Poor & 1009 & 80.5 \\
\hline Good & 244 & 19.5 \\
\hline \multicolumn{3}{|l|}{ Access to health facility } \\
\hline Not easy & 485 & 38.7 \\
\hline Easy & 768 & 61.3 \\
\hline Distance to health facility & $\mathrm{N} / \mathrm{A}$ & $\mathrm{N} / \mathrm{A}$ \\
\hline
\end{tabular}

In this study, we used Pearson's Chi-square statistic to assess the relationship between each socio-environmental determinant and perceived exposure to onchocerciasis. Based on the chisquared statistic reported for migration status, housing quality and self-rated ability to handle personal crisis, we failed to reject the hypothesis that these variables and perceived exposure to onchocerciasis are independent. The magnitude of Cramer's V statistic between perceived exposure to onchocerciasis and most variables were less than 0.3 , except urbanicity wealth 
index and ethnicity indicating that the associations between most variables and perceived exposure to onchocerciasis was not strong. Results of associations between socio-environmental determinants and perceived exposure to onchocerciasis are presented in Table 2.

Table 2: Measure of association between perceived exposure to onchocerciasis and socioenvironmental determinants

\begin{tabular}{|c|c|c|c|c|}
\hline \multicolumn{5}{|l|}{ Variables } \\
\hline & No $(\%)$ & Yes $(\%)$ & & \\
\hline Urbanicity wealth index & & & Pearson $\operatorname{chi} 2(5)=169.0464$ & $\operatorname{Pr}=\mathbf{0 . 0 0 0}$ \\
\hline Rural poor & 51.3 & 48.7 & Cramér's V $=0.3673$ & \\
\hline Rural middle & 54.7 & 45.3 & & \\
\hline Rural rich & 66.7 & 33.3 & & \\
\hline Urban poor & 82.5 & 17.5 & & \\
\hline Urban middle & 90.2 & 9.8 & & \\
\hline Urban rich & 84.2 & 15.8 & & \\
\hline Educational status & & & Pearson chi2 $(3)=68.7287$ & $\operatorname{Pr}=\mathbf{0 . 0 0 0}$ \\
\hline No education & 57.9 & 42.1 & Cramér's V $=0.2342$ & \\
\hline Primary & 63.5 & 36.5 & & \\
\hline Secondary & 80.8 & 19.2 & & \\
\hline Tertiary & 86.9 & 13.1 & & \\
\hline Self-rated health status & & & Pearson chi2 $(2)=23.2496$ & $\operatorname{Pr}=\mathbf{0 . 0 0 0}$ \\
\hline Poor & 69.6 & 30.4 & Cramér's V $=0.1362$ & \\
\hline Fair & 69.1 & 30.9 & & \\
\hline Good & 85.8 & 14.2 & & \\
\hline Self-rated ability to handle personal crisis & & & Pearson chi2 $(1)=2.4160$ & $\operatorname{Pr}=0.120$ \\
\hline Poor & 73.4 & 26.6 & Cramér's V = 0.0439 & \\
\hline Good & 69.3 & 30.7 & & \\
\hline Self-rated ability to handle work pressure & & & Pearson chi2 $(1)=6.1048$ & $\operatorname{Pr}=\mathbf{0 . 0 1 3}$ \\
\hline Poor & 68.1 & 31.9 & Cramér's V $=-0.0698$ & \\
\hline Good & 74.5 & 25.5 & & \\
\hline Reliance on social network & & & Pearson chi2 $(1)=28.1840$ & $\operatorname{Pr}=\mathbf{0 . 0 0 0}$ \\
\hline No & 69.3 & 30.7 & Cramér's V $=-0.1500$ & \\
\hline Yes & 89.2 & 10.8 & & \\
\hline Migration status & & & Pearson chi2 $(1)=0.0006$ & $\operatorname{Pr}=0.981$ \\
\hline Within coastal areas & 71.9 & 28.1 & Cramér's V $=0.0007$ & \\
\hline From inland to coastal areas & 71.8 & 28.2 & & \\
\hline Ethnicity & & & Pearson chi2 $(2)=170.0741$ & $1 \operatorname{Pr}=\mathbf{0 . 0 0 0}$ \\
\hline Zaramo & 99.2 & 0.8 & Cramér's V = 0.3684 & \\
\hline Sambaa & 37.4 & 62.6 & & \\
\hline Others & 69.4 & 30.6 & & \\
\hline Housing quality & & & Pearson chi2 $(1)=2.8020$ & $\operatorname{Pr}=0.094$ \\
\hline Poor & 70.9 & 29.1 & Cramér's V $=-0.0473$ & \\
\hline
\end{tabular}


Good

Access to health facility

Not easy

Easy
76.2

57.9

80.7
23.8

Pearson $\operatorname{chi} 2(1)=76.4389 \operatorname{Pr}=\mathbf{0 . 0 0 0}$

42.1 Cramér's V $=-0.2470$

At the bivariate level, urbanicity wealth index, education, ethnicity, age, access and distance to health facility, self-rated health status, reliance on social support and migration status were all significant predictors of perceived exposure to onchocerciasis. Residents in urban areas irrespective of their wealth status (urban poor, urban middle and urban rich) were less likely to report perceived exposure to onchocerciasis. Likewise, individuals who had attained secondary $(\mathrm{OR}=0.53, \mathrm{p}<0.001)$ or tertiary education $(\mathrm{OR}=0.43, \mathrm{p}<0.001)$, had easy access to health facility $(\mathrm{OR}=0.53, \mathrm{p}<0.001)$, and rated their health status as good $(\mathrm{OR}=0.61, \mathrm{p}<0.001)$ were all less likely to report perceived exposure to onchocerciasis. Those who had social network $(\mathrm{OR}=0.53$, $\mathrm{p}<0001$ ) were less likely to report being in an onchocerciasis endemic environment relative to those with no social support. Conversely, respondents from the Sambaa $(O R=10.26, p<0.001)$ and other ethnic groups $(\mathrm{OR}=4.06, \mathrm{p}<0.001)$ were more likely to be report perceived exposure to onchocerciasis relative to those belonging to the Zaramo ethnicity. Individuals in the age categories 36-50 $(\mathrm{OR}=1.54, \mathrm{p}<0.001), 51-65(\mathrm{OR}=1.57, \mathrm{p}<0.001)$ and 66 or older $(\mathrm{OR}=1.41$, $\mathrm{p}<0.001)$ were all more likely to report perceived exposure to onchocerciasis compared with those in the 18-35 age group.

Table 3: Bivariate relationship between perceived exposure to onchocerciasis and socioenvironmental determinants in coastal Tanzania

\begin{tabular}{|c|c|c|c|c|c|}
\hline \multirow[b]{2}{*}{ Behaviour } & \multirow[t]{2}{*}{ OR } & \multirow[t]{2}{*}{ Robust SE } & \multirow[t]{2}{*}{ p-value } & \multicolumn{2}{|c|}{ Confidence Interval } \\
\hline & & & & & \\
\hline \multicolumn{6}{|c|}{ Urbanicity wealth index (Ref: Rural poor) } \\
\hline Rural middle & 0.91 & 0.16 & 0.589 & 0.65 & 1.28 \\
\hline Rural rich & 0.66 & 0.20 & 0.175 & 0.36 & 1.21 \\
\hline Urban poor & 0.41 & 0.04 & 0.000 & 0.34 & 0.50 \\
\hline Urban middle & 0.31 & 0.03 & 0.000 & 0.25 & 0.39 \\
\hline Urban rich & 0.39 & 0.04 & 0.000 & 0.32 & 0.48 \\
\hline \multicolumn{6}{|c|}{ Educational status (Ref: No education) } \\
\hline Primary & 0.86 & 0.12 & 0.290 & 0.65 & 1.13 \\
\hline Secondary & 0.53 & 0.08 & 0.000 & 0.39 & 0.70 \\
\hline Tertiary & 0.43 & 0.07 & 0.000 & 0.31 & 0.58 \\
\hline \multicolumn{6}{|c|}{ Self-rated health status (Ref: Poor) } \\
\hline Fair & 1.01 & 0.10 & 0.888 & 0.83 & 1.24 \\
\hline Good & 0.61 & 0.08 & 0.000 & 0.48 & 0.78 \\
\hline \multicolumn{6}{|c|}{ Self-rated ability to handle personal crisis (Ref: Poor) } \\
\hline Good & 1.12 & 0.08 & 0.123 & 0.97 & 1.30 \\
\hline \multicolumn{6}{|c|}{ Self-rated ability to handle work crisis (Ref: Poor) } \\
\hline Good & 0.84 & 0.06 & 0.014 & 0.72 & 0.96 \\
\hline
\end{tabular}




\begin{tabular}{lccccc} 
Yes & 0.53 & 0.06 & $\mathbf{0 . 0 0 0}$ & 0.43 & 0.66 \\
$\begin{array}{l}\text { Migration (Ref: Within coastal areas) } \\
\text { From inland to coastal areas }\end{array}$ & 1.27 & 0.09 & $\mathbf{0 . 0 0 1}$ & 1.10 & 1.46 \\
$\begin{array}{l}\text { Population } \\
\text { Age (Ref: 18-35) }\end{array}$ & & & & \\
36-50 & 1.54 & 0.13 & $\mathbf{0 . 0 0 0}$ & 1.30 & 1.82 \\
$51-65$ & 1.57 & 0.15 & $\mathbf{0 . 0 0 0}$ & 1.31 & 1.89 \\
$>65$ & 1.41 & 0.21 & $\mathbf{0 . 0 2 4}$ & 1.04 & 1.89 \\
Gender (Ref: Male) & & & & & \\
$\begin{array}{l}\text { Female } \\
\text { Ethnicity (Ref: Zaramo) }\end{array}$ & 1.08 & 0.08 & 0.300 & 0.94 & 1.24 \\
Sambaa & 10.26 & 2.11 & $\mathbf{0 . 0 0 0}$ & 6.86 & 15.36 \\
$\begin{array}{l}\text { Others } \\
\text { Habitat }\end{array}$ & 4.06 & 0.62 & $\mathbf{0 . 0 0 0}$ & 3.01 & 5.47 \\
$\begin{array}{l}\text { Household quality (Ref: Poor) } \\
\text { Good } \\
\text { Access to health facility (Ref: Not easy) }\end{array}$ & & & & & \\
Easy & 0.86 & 0.08 & 0.087 & 0.72 & 1.02 \\
$\begin{array}{l}\text { Distance to health facility (Ref: Short) } \\
\text { Long }\end{array}$ & 0.53 & 0.04 & $\mathbf{0 . 0 0 0}$ & 0.45 & 0.61 \\
\hline
\end{tabular}

Unadjusted multivariate model

The results of the unadjusted and adjusted multivariate analysis are displayed in Table 4 and Table 5, respectively. Table 4 presents the unadjusted results showing the independent effects of each set of the population, behavioural and habitat factors on perceived exposure to onchocerciasis. As presented in Table 4, all the population factors were significantly associated with perceived exposure to onchocerciasis. For instance, women (OR=1.18, CI: 1.01-1.39), and those from the Sambaa ethnicity (OR=10.69, CI: 6.77-16.89) had significantly higher odds of reporting that they were exposed to onchocerciasis. In terms of the behavioural factors, only those with tertiary education $(\mathrm{OR}=0.63$. $\mathrm{CI}: 0.42-0.94)$ had lower odds of reporting exposure to onchocerciasis compared with their counterparts without formal education. Individuals who migrated from inland area $(\mathrm{OR}=1.22, \mathrm{CI}: 1.05-1.43)$ were more likely to report perceived exposure to onchocerciasis relative to those who migrated from coastal areas. In the habitat model, individuals who lived far from a health facility $(\mathrm{OR}=1.37, \mathrm{CI}$ : 1.25-1.49) were more likely to report perceived exposure to onchocerciasis compared with those who reported living in proximity to a health facility. Additionally, residents who had access to a health facility $(\mathrm{OR}=0.83$, CI: 0.69-0.99) were less likely to report perceived exposure to onchocerciasis. 
Table 4: Multivariate relationship between perceived exposure to onchocerciasis and socioenvironmental determinants in coastal Tanzania (unadjusted)

\begin{tabular}{|c|c|c|c|c|c|}
\hline & OR & Robust SE & $\mathrm{p}$-value & \multicolumn{2}{|c|}{ Confidence Interval } \\
\hline \multicolumn{6}{|l|}{ Behaviour } \\
\hline \multicolumn{6}{|c|}{ Urbanicity wealth index (Ref: Rural poor) } \\
\hline rural middle & 0.93 & 0.17 & 0.706 & 0.66 & 1.33 \\
\hline rural rich & 0.93 & 0.34 & 0.849 & 0.45 & 1.92 \\
\hline urban poor & 0.40 & 0.04 & 0.000 & 0.33 & 0.50 \\
\hline urban middle & 0.33 & 0.04 & 0.000 & 0.26 & 0.43 \\
\hline urban rich & 0.52 & 0.09 & 0.000 & 0.38 & 0.72 \\
\hline \multicolumn{6}{|c|}{ Educational status (Ref: No education) } \\
\hline Primary & 0.92 & 0.13 & 0.585 & 0.69 & 1.23 \\
\hline Secondary & 0.77 & 0.12 & 0.103 & 0.56 & 1.05 \\
\hline Tertiary & 0.63 & 0.13 & 0.023 & 0.42 & 0.94 \\
\hline \multicolumn{6}{|c|}{ Self-rated health status (Ref: Poor) } \\
\hline Fair & 1.09 & 0.11 & 0.420 & 0.89 & 1.33 \\
\hline Good & 0.93 & 0.14 & 0.637 & 0.69 & 1.25 \\
\hline \multicolumn{6}{|c|}{ Self-rated ability to handle personal crisis (Ref: Poor) } \\
\hline Good & 1.51 & 0.13 & 0.000 & 1.27 & 1.80 \\
\hline \multicolumn{6}{|c|}{ Reliance on social network (Ref: No) } \\
\hline Yes & 0.75 & 0.09 & 0.018 & 0.59 & 0.95 \\
\hline \multicolumn{6}{|l|}{ Migration (Ref: Coastal areas) } \\
\hline From inland to coastal areas & 1.22 & 0.10 & 0.011 & 1.05 & 1.43 \\
\hline \multicolumn{6}{|l|}{ Population } \\
\hline \multicolumn{6}{|l|}{ Age (Ref: 18-35) } \\
\hline $36-50$ & 1.63 & 0.16 & 0.000 & 1.34 & 1.99 \\
\hline $51-65$ & 1.79 & 0.21 & 0.000 & 1.42 & 2.24 \\
\hline$>65$ & 1.64 & 0.27 & 0.003 & 1.18 & 2.28 \\
\hline \multicolumn{6}{|l|}{ Gender (Ref: Male) } \\
\hline Female & 1.18 & 0.10 & 0.043 & 1.01 & 1.39 \\
\hline \multicolumn{6}{|l|}{ Ethnicity (Ref: Zaramo) } \\
\hline Sambaa & 10.69 & 2.49 & 0.000 & 6.77 & 16.89 \\
\hline Others & 4.20 & 0.77 & 0.000 & 2.92 & 6.02 \\
\hline \multicolumn{6}{|l|}{ Habitat } \\
\hline \multicolumn{6}{|l|}{ Household quality (Ref: Poor) } \\
\hline Good & 1.03 & 0.10 & 0.732 & 0.86 & 1.24 \\
\hline \multicolumn{6}{|c|}{ Access to health facility (Ref: Not easy) } \\
\hline Easy & 0.83 & 0.08 & 0.042 & 0.69 & 0.99 \\
\hline \multicolumn{6}{|c|}{ Distance to health facility (Ref: Short) } \\
\hline Long & 1.37 & 0.06 & 0.000 & 1.25 & 1.49 \\
\hline
\end{tabular}




\section{Adjusted multivariate model}

After adjusting for theoretically relevant factors in Models 2 and 3 in Table 5, results still established various socio-environmental determinants as important predictors of report perceived exposure to onchocerciasis in coastal Tanzania. For instance, results in Model 1 (population model) showed that individuals from other ethnic groups (OR=4.20, CI: 2.92-6.02) were more likely to report perceived exposure to onchocerciasis compared with their Zaramo counterparts. Likewise, residents from the Sambaa ethnic group (OR=10.69, CI: 6.77-16.89) were far more likely to report perceived exposure to onchocerciasis compared with those from the Zaramo ethnic group. Furthermore, individuals in all age groups 36-50 (OR=1.63, CI: 1.341.99), 51-65 (OR=1.79, CI: 1.42-2.24) and 66 or older (OR=1.64, CI: 1.18-2.28) had higher odds of reporting being exposed to onchocerciasis compared with those in the 18-35 age groups. When behavioural factors were accounted for in the original population model (Model 2 ), the odds ratio for the Sambaa ethnic group increased by $88 \%$ and further reduced by $11 \%$ when habitat factors were accounted for in the final multivariate model (Model 3). Individuals in age categories (36-50) and (51-65) were significantly more likely to report perceived exposure to onchocerciasis. However, the relationship between age category $(>65)$ and selfreported exposure to onchocerciasis disappeared when behavioural and habitat factors were adjusted for in Model 3. Respondents who had attained secondary (OR=0.51, CI: $0.30-0.85)$ or tertiary education $(\mathrm{OR}=0.37, \mathrm{CI}$ : $0.20-0.65)$ had lower odds of reporting perception of exposure to onchocerciasis compared with their counterparts without formal education. Likewise, when behavioural and habitat factors were taken into account in the final model, individuals who had attained secondary or tertiary education were $58 \%$ and $40 \%$ respectively, less likely to report perceived exposure to onchocerciasis compared with their uneducated counterparts. 
Table 5: Negative log-log model showing the multivariate relationship between perceived exposure to onchocerciasis and socioenvironmental determinants in coastal Tanzania (adjusted)

\begin{tabular}{|c|c|c|c|c|c|c|c|c|c|c|c|c|c|c|c|}
\hline & \multicolumn{5}{|c|}{ Model 1} & \multicolumn{5}{|c|}{ Model 2} & \multicolumn{5}{|c|}{ Model 3} \\
\hline & \multicolumn{5}{|c|}{ POPULATION } & \multicolumn{5}{|c|}{ POPULATION + BEHAVIOURAL } & \multicolumn{5}{|c|}{ POPULATION + BEHAVIOURAL + HABITAT } \\
\hline & OR & $\begin{array}{c}\text { Robust } \\
\text { SE }\end{array}$ & $\begin{array}{c}\mathrm{p}- \\
\text { value }\end{array}$ & \multicolumn{2}{|c|}{$\begin{array}{c}\text { Confidence } \\
\text { Interval } \\
\end{array}$} & OR & $\begin{array}{c}\text { Robust } \\
\text { SE }\end{array}$ & $\begin{array}{l}\mathrm{p}- \\
\text { value }\end{array}$ & \multicolumn{2}{|c|}{$\begin{array}{l}\text { Confidence } \\
\text { Interval }\end{array}$} & OR & $\begin{array}{c}\text { Robust } \\
\text { SE }\end{array}$ & $\begin{array}{c}\text { p- } \\
\text { value }\end{array}$ & \multicolumn{2}{|c|}{$\begin{array}{c}\text { Confidence } \\
\text { Interval }\end{array}$} \\
\hline $36-50$ & 1.63 & 0.16 & 0.000 & 1.34 & 1.99 & 1.52 & 0.16 & 0.000 & 1.23 & 1.87 & 1.53 & 0.17 & 0.000 & 1.23 & 1.90 \\
\hline $51-65$ & 1.79 & 0.21 & 0.000 & 1.42 & 2.24 & 1.68 & 0.21 & 0.000 & 1.32 & 2.13 & 1.67 & 0.21 & 0.000 & 1.30 & 2.13 \\
\hline$>65$ & 1.64 & 0.27 & 0.003 & 1.18 & 2.28 & 1.25 & 0.24 & 0.245 & 0.86 & 1.83 & 1.33 & 0.27 & 0.160 & 0.89 & 1.98 \\
\hline \multicolumn{16}{|c|}{ Gender (Ref: Male) } \\
\hline Female & 1.18 & 0.10 & 0.043 & 1.01 & 1.39 & 1.17 & 0.11 & 0.093 & 0.97 & 1.40 & 1.15 & 0.11 & 0.143 & 0.95 & 1.38 \\
\hline \multicolumn{16}{|c|}{ Ethnicity (Ref: Zaramo) } \\
\hline Sambaa & 10.69 & 2.49 & 0.000 & 6.77 & 16.89 & 20.09 & 5.09 & 0.000 & 12.23 & 33.01 & 17.94 & 4.60 & 0.000 & 10.85 & 29.65 \\
\hline Others & 4.20 & 0.77 & 0.000 & 2.92 & 6.02 & 8.87 & 1.96 & 0.000 & 5.75 & 13.68 & 8.16 & 1.79 & 0.000 & 5.31 & 12.53 \\
\hline \multicolumn{16}{|c|}{ Urbanicity wealth index (Ref: Rural poor) } \\
\hline rural middle & & & & & & 0.91 & 0.19 & 0.653 & 0.61 & 1.36 & 0.95 & 0.20 & 0.799 & 0.63 & 1.44 \\
\hline rural rich & & & & & & 0.90 & 0.37 & 0.793 & 0.40 & 1.99 & 0.96 & 0.41 & 0.932 & 0.42 & 2.23 \\
\hline urban poor & & & & & & 0.27 & 0.04 & 0.000 & 0.21 & 0.36 & 0.31 & 0.05 & 0.000 & 0.24 & 0.42 \\
\hline urban middle & & & & & & 0.23 & 0.04 & 0.000 & 0.16 & 0.33 & 0.26 & 0.05 & 0.000 & 0.18 & 0.37 \\
\hline urban rich & & & & & & 0.38 & 0.08 & 0.000 & 0.25 & 0.56 & 0.41 & 0.08 & 0.000 & 0.27 & 0.61 \\
\hline \multicolumn{16}{|c|}{ Educational Status (Ref: No education) } \\
\hline Primary & & & & & & 0.76 & 0.19 & 0.277 & 0.47 & 1.24 & 0.87 & 0.20 & 0.554 & 0.56 & 1.37 \\
\hline Secondary & & & & & & 0.51 & 0.14 & 0.011 & 0.30 & 0.85 & 0.58 & 0.14 & 0.029 & 0.36 & 0.95 \\
\hline Tertiary & & & & & & 0.37 & 0.11 & 0.001 & 0.20 & 0.65 & 0.40 & 0.11 & 0.001 & 0.23 & 0.70 \\
\hline \multicolumn{16}{|c|}{ Self-rated health status (Ref: Poor) } \\
\hline Fair & & & & & & 1.23 & 0.17 & 0.125 & 0.94 & 1.61 & 1.35 & 0.20 & 0.042 & 1.01 & 1.81 \\
\hline Good & & & & & & 1.22 & 0.22 & 0.264 & 0.86 & 1.74 & 1.30 & 0.24 & 0.170 & 0.89 & 1.87 \\
\hline \multicolumn{16}{|c|}{ Self-rated ability to handle personal crisis (Ref: Poor) } \\
\hline Good & & & & & & 1.56 & 0.16 & 0.000 & 1.28 & 1.91 & 1.56 & 0.16 & 0.000 & 1.27 & 1.92 \\
\hline
\end{tabular}


Yes

Migration status Ref: within coastal areas)

From inland to coastal areas

Housing quality (Ref: Poor)

Good

Access to health facility (Ref: Not easy)

Easy

Distance to health facility (Ref: Short)

\begin{tabular}{|c|c|c|c|c|c|c|c|c|c|}
\hline 0.73 & 0.10 & 0.023 & 0.55 & 0.96 & 0.71 & 0.10 & 0.013 & 0.54 & 0.93 \\
\hline \multirow[t]{4}{*}{1.31} & 0.15 & 0.019 & 1.05 & 1.63 & 1.30 & 0.15 & 0.022 & 1.04 & 1.63 \\
\hline & & & & & 1.23 & 0.15 & 0.087 & 0.97 & 1.56 \\
\hline & & & & & 1.17 & 0.13 & 0.162 & 0.94 & 1.44 \\
\hline & & & & & 1.25 & 0.06 & 0.000 & 1.14 & 1.38 \\
\hline
\end{tabular}


Results, as presented in Model 2 (Table 5), indicate that in terms of urbanicity wealth index, rural residence regardless of wealth status was not a statistically significant predictor of perceived exposure to onchocerciasis. However, residents in urban locations irrespective of wealth status were less likely to report living in onchocerciasis endemic areas. For instance, the urban poor, urban middle and urban rich were $73 \%, 77 \%, 62 \%$ respectively, less likely to report perceived exposure to onchocerciasis. These percentages increased by $15 \%, 13 \%, 8 \%$ respectively, when habitat factors were adjusted for in the final model. In our study, fair selfrated health status $(\mathrm{OR}=1.35, \mathrm{CI}$ : 1.01-1.81) was a significant predictor of perceived exposure to onchocerciasis when population, behaviour and habitat factors were accounted for in the final adjusted model. Also, after adjusting for the effects of the selected behavioural and habitat factors in Models 2-3, individuals who rated their ability to handle personal crisis as good were $56 \%$ more likely to report perceived exposure to onchocerciasis compared with those whose ability to deal with personal crisis was poor. Participants who migrated from inland to coastal areas (OR=1.31, CI: 1.05-1.63) had higher odds of reporting perceived exposure to onchocerciasis compared with those who migrated within coastal areas. Additionally, individuals who live farther from health facilities were significantly more likely to report perceived exposure to onchocerciasis compared with those who live in proximity to a health facility in the joint population, behavioural and habitat model (Model 3). Figure 3 illustrates the multivariate relationship between the socio-environmental factors and perception of onchocerciasis.

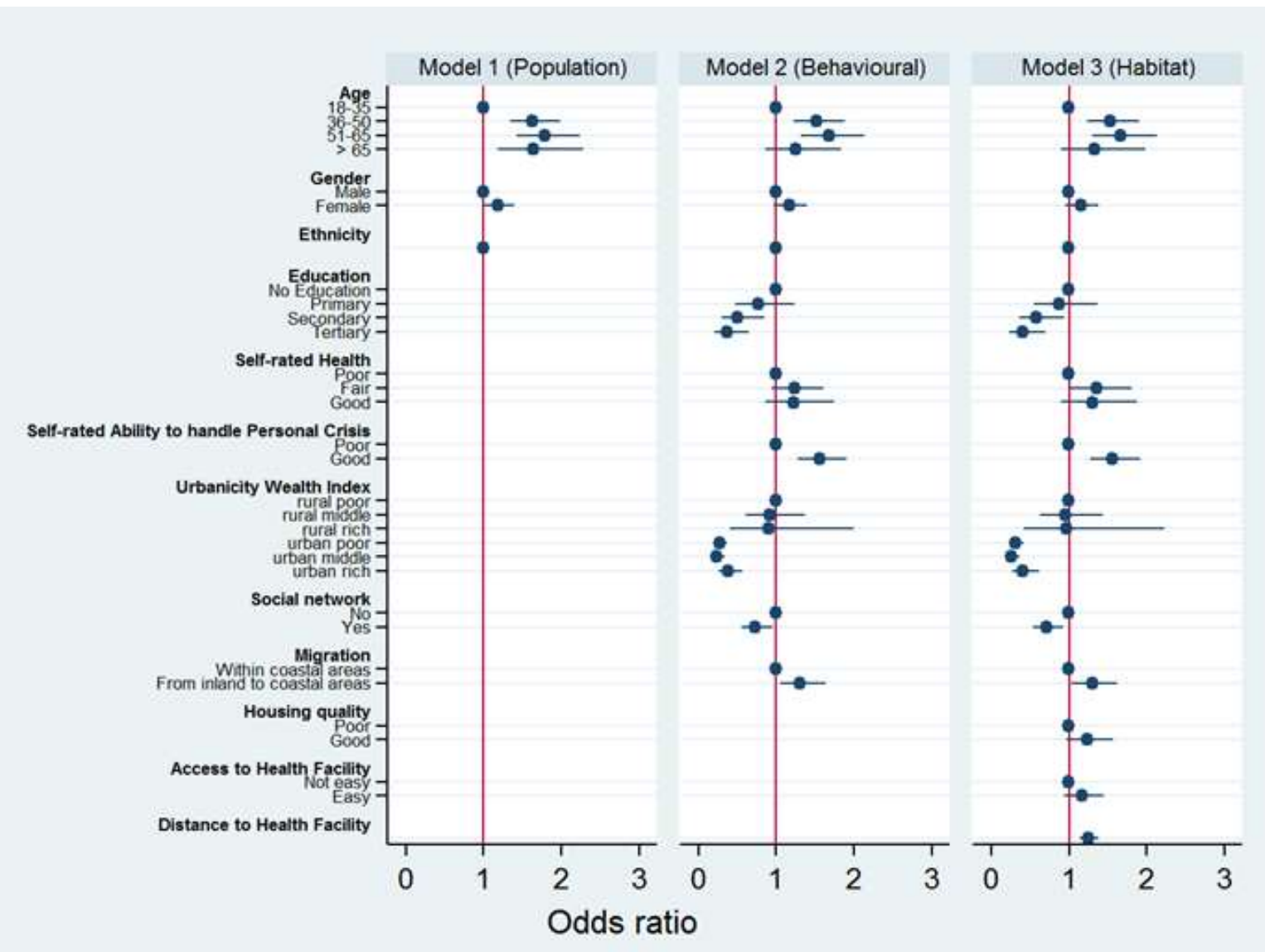

Figure 3: Graphical representation of estimated odds ratios 


\section{Discussion}

In this paper, we examined the relationship between socio-environmental determinants (population, behavioural and habitat factors) and perception of onchocerciasis exposure among coastal dwellers in Tanzania. Understanding the independent effects of these determinants on public perception of exposure to onchocerciasis is very important when it comes to controlling the spread of neglected tropical diseases (NTDs) in sub-Saharan Africa. Our findings indicate that urbanicity wealth index was an important predictor of perceived exposure to onchocerciasis and that urban residents irrespective of their wealth status were less likely to report perceived exposure to onchocerciasis suggesting geographical disparities in exposure to the disease. This result supports emerging belief in an urban advantage - the notion that urban dwellers are invariably better off compared with rural residents (UNICEF, 2012) in terms of health status. The urban areas of Tanga and Dar es Salaam are definitely non-endemic as there are no Simulium vectors in these urban areas and therefore no transmission. In the Tanga and Pwani regions in Tanzania, onchocerciasis used to be endemic and a focus in the Usambara Mountains in Muheza district and a narrow strip at the western border of Bagamoyo district. In the rest of the study districts onchocerciasis is not endemic. Also, urban residence might confer an advantage including more access to the necessities of life such as health care, quality housing opportunities and education (Vlahov, Galea, \& Freudenberg, 2005). On the contrary, rural dwellers confront several barriers to health care to a far greater degree than their urban counterparts due to geographical isolation (Kadobera et al., 2012), especially in developing countries. Tanzania is a predominantly rural country and health status of rural residents continues on average to be worse than that of urban populations (Kruk et al., 2009; World Bank, 2003).

Onchocerciasis is a rural disease because environmental conditions in urban environments do not allow for vector breeding. Hence, except for one suburb of Brazzaville next to huge rapids in the River Congo, all other 36 million people at risk of onchocerciasis in Africa live in rural areas. Affected communities are generally poor farming communities that are often located in isolated areas far from health facilities and education. Hence, distance to health facility and educational levels are not determinants of individual exposure but a characteristic of onchocerciasis endemic areas.

Geographic accessibility (i.e., physical proximity) to health facility may present an important barrier to access to health services especially in rural areas due to limited health facilities, cost and transportation problems experienced in these areas (Awoyemi, Obayelu, \& Opaluwa, 2011; Kadobera et al., 2012). Our findings suggest that perceived exposure to onchocerciasis was significantly higher among respondents who reported travelling long distance to health facilities. This result is consistent with studies that have found long travel times and greater distances to health care facilities as having negative influence on health care services utilization (Awoyemi, Obayelu, \& Opaluwa, 2011; Buor, 2003).

Results of this study established that educational attainment, ethnicity, social support, and migration status are also significant predictors of perceived exposure to onchocerciasis. The emergence of education as a protective factor against onchocerciasis is consistent with the work of Weldegebreal et al. (2014) and York et al. (2015). It is a well-established fact that education 
plays a vital role in shaping cultures, norms, opinions, and attitudes regarding awareness and potential disease prevention. In our study, individuals with secondary of higher education had less likelihood of living in an onchocerciasis endemic environment. This is important to note since $55 \%$ of respondents in this study have primary or no education and had no idea of how the disease is transmitted suggesting that the attempt to improve knowledge on the causes and perceptions of onchocerciasis risk must include strategies aimed at increasing awareness of the disease through existing community-based disease control programs. It would seem reasonable to hypothesize that factors that affect knowledge of onchocerciasis may also systematically affect self-reporting of onchocerciasis. To wit, some of the protective variables - urbanicity, education, proximity/access to health may be hypothesized to actually protect against onchocerciasis exposure consistent with our observed association with reduced reporting of exposure but one could also hypothesize that they also/instead act to support more accurate perceptions and beliefs about onchocerciasis exposure and may correct for inaccurate overinflation of perceived exposure among those who lack these factors. Several pertinent questions, therefore, arise. Are these factors epidemiologically protective or epistemologically pseudoprotective? For instance, a person who believes onchocerciasis is an STI and is not corrected in this belief by a rich/dense/higher quality urban network of informal knowledge, personal education, or proximity of health care facilities that promulgate true knowledge about onchocerciasis within those local networks may have reason to mistakenly report onchocerciasis exposure more than a person who has these factors to correct their mistaken overestimation of exposure.

Generally, health education about the endemicity of the disease leads to awareness and provides the necessary information for change in beliefs, attitudes and behaviour (Karunamoorthi, Kassa, \& Endale, 2010; Okwa, Olusola, \& Adelani, 2009). Consistent with our findings, other studies have shown that people with little or no formal education tend to believe in a wide range of misconceptions about the causes and method of transmission of onchocerciasis (Yirga et al., 2008). In a study conducted in Cameroon, Hewlett et al. (1996) found that majority of respondents believed onchocerciasis could be transmitted sexually. This concurs with the results from an Ethiopian study, which found that only $11 \%$ of the subjects knew about the etiology of the disease (Weldegebreal et al., 2014). Contrary to these findings, Lakwo and Gasarasi (2006) found that high proportion $(66 \%)$ of respondents in Southwest Tanzania had heard of onchocerciasis.

Due to the cultural differences among Tanzanians, there are potentially huge ethnic variations in exposure to onchocerciasis in coastal areas of the country. Our findings suggest that those from the Sambaa and other ethnic groups were far more likely to report increased risk perception to onchocerciasis compared with the Zaramo. This is understandable given that onchocerciasis is most common in the region between Lake Nyasa and the Usambara mountains (Muro and Mziray, 1990) where the Sambaa originate. Individuals of same ethnic groups tend to live in proximity to one another indicating the clustering of infection within communities and the importance of social connectivity in disease transmission (Giebultowicz et al., 2011). This inference about clustering and rich connectivity among individuals of ethnic groups as opposed to between ethnic groups may not just support or be consistent with the "importance of social connectivity in disease transmission" but may also support or be consistent with the importance of social connectivity with transmission of beliefs about disease exposure. Yet, we do not know 
if there are ethnicity specific patterns of belief about onchocerciasis that may systematically affect beliefs about one's exposure. Our result shows that not only does onchocerciasis cluster spatially, but it can also cluster within social networks as it is transmitted through the local environment. The relative contribution of social support indicates that individuals who perceive their families as supportive are less likely to report risk perception to onchocerciasis. The availability of appropriate social support network is important for social inclusion especially under stressful life events such as exposure to onchocerciasis.

\section{Limitations of the study}

There are some limitations to borne in mind while interpreting the findings of this study. First, cause-effect relationships could not be established due to the cross-sectional nature of the data. Also, most of the socio-environmental determinants were based on self-reports, which is subject to recall bias. To address this, respondents were only asked to report on events within the previous year.

\section{Conclusion}

Onchocerciasis is a disease of poverty with severe socio-economic consequences. This disease among other NTDs is most prevalent in the poorest populations in the world with sub-Saharan Africa bearing the biggest burden. The key to controlling onchocerciasis lies in understanding the geographical distribution and socio-environmental determinants of the disease. Findings of this study clearly established that socio-environmental determinants such as population, behavioural and habitat factors are significant predictors of self-reported exposure to onchocerciasis. This study revealed the need for both government and non-governmental organizations in Tanzania to increase the awareness and prevention of onchocerciasis in the country through health education campaigns at the community level regarding transmission, appropriate prevention techniques and address barriers to accessing health care services especially, in rural areas. Given that ethnicity was a very important determinant, there is need for policy makers to address place specific factors that predispose certain ethnic groups to the disease. Similarly, since relative well-being contributed significantly to disparities in perceived exposure to onchocerciasis, social support systems are required to address vulnerability of certain populations to the disease.

\section{Acknowledgement}

Research funding from the Major Collaborative Research Initiative (MCRI) of the Social Sciences and Humanities Research Council (SSHRC) Canada is duly acknowledged. This research is part of the initiative "the Indian Ocean World: The Making of the First Global Economy in the Context of Human Environment Interaction". The funders were not involved in study design, data collection and analysis, decision to publish, or preparation of the manuscript. 


\section{References}

Amazigo, U. (2008). The African Programme for Onchocerciasis Control (APOC). Ann Trop Med Parasitol 102 Suppl 1: 19-22.

Armah, F. A., Luginaah, I., Hambati, H., Chuenpagdee, R., \& Campbell, G. (2015a). Assessing barriers to adaptation to climate change in coastal Tanzania: Does where you live matter? Population and Environment, 37(2), 231-263.

Armah, F. A., Luginaah, I., Yengoh, G. T., Hambati, H., Chuenpagdee, R., \& Campbell, G. (2015b). Analyzing the relationship between objective-subjective health status and public perception of climate change as a human health risk in coastal Tanzania. Human and Ecological Risk Assessment: An International Journal 21(7), 1936-1959.

Boamah, S. A., Armah, F. A., Luginaah, I., Hambati, H., Chuenpagdee, R., \& Campbell, G. (2017). Evaluating the complex interactions between malaria and cholera prevalence, neglected tropical disease comorbidities, and community perception of health risks of climate change. Journal of Human Behavior in the Social Environment, 27(7), 714-732.

Brewer, N. T., Chapman, G. B., Gibbons, F. X., Gerrard, M., McCaul, K. D., \& Weinstein, N. D. (2007). Meta-analysis of the relationship between risk perception and health behavior: the example of vaccination. Health Psychology, 26(2), 136.

APOC (2009). Informal consultation on eliminationof onchocerciasis transmission with current tools inAfrica - "shrinking the map". Ouagadougou, World Health Organisation/African Programme for Onchocerciasis Control.

Awolola, T.S., Manafa, O.U., Rotimi, O.O, Ogunrinade, A.F. (2000). Knowledge and beliefs about causes, transmission, treatment and control of human onchocerciasis in rural communities in south western Nigeria. Acta Trop, 76(3), 247-51.

Buor, D. (2005). Determinants of utilisation of health services by women in rural and urban areas in Ghana. GeoJournal, 61(1), 89-102.

Coffeng, L. E., Pion, S. D., O'Hanlon, S., Cousens, S., Abiose, A. O., Fischer, P. U., ... \& Boussinesq, M. (2013). Onchocerciasis: the pre-control association between prevalence of palpable nodules and skin microfilariae. PLoS neglected tropical diseases, 7(4), e2168.

Curtis, S. E., \& Oven, K. J. (2012). Geographies of health and climate change. Progress in Human Geography, 36(5), 654-666.

Ferrer, R., \& Klein, W. M. (2015). Risk perceptions and health behavior. Current Opinion in Psychology, 5, 85-89.

Francis, J., \& Bryceson, I. (2001). Tanzanian coastal and marine resources: some examples illustrating questions of sustainable use. Chapter, 4, 76-102.

Giebultowicz, S., Ali, M., Yunus, M., \& Emch, M. (2011). A comparison of spatial and social clustering of cholera in Matlab, Bangladesh. Health \& place, 17(2), 490-497.

Granado, S., Manderson, L., Obrist, B., Tanner, M. (2011). Appropriating "malaria": local responses to malaria treatment and prevention in Abidjan, Cote d'Ivoire. Medical Anthropology, 30(1), 102-121. 
Hotez, P. J., \& Kamath, A. (2009). Neglected tropical diseases in sub-Saharan Africa: review of their prevalence, distribution, and disease burden. PLoS neglected tropical diseases, 3(8), 412.

Hotez, P.J., Molyneux, D.H., Fenwick, A., Kumaresan, J., Ehrlich Sachs, S., Sachs, J.D. and Savioli, L. (2007). Current Concepts: Control of Neglected Tropical Diseases. New England Journal of Medicine 357(10):1018-1027. http://www.nejm.org/doi/pdf/10.1056/NEJMra064142

Kadobera, D., Sartorius, B., Masanja, H., Mathew, A., Waiswa, P. (2012). The effect of distance to formal health facility on childhood mortality in rural Tanzania, 2005-2007. Global Health Action, 5, 1-9.

Karunamoorthi, K., Kassa, E., \& Endale, A. (2010). Knowledge and beliefs about onchocerciasis among rural inhabitants in an endemic area of Ethiopia. International health, 2(1), 59-64.

Lakwo, T. L., \& Gasarasi, D. B. (2006). Non-adherence to community directed treatment with ivermectin for onchocerciasis control in Rungwe district, southwest Tanzania. East African medical journal, 83(6), 326-332.

Mackenzie, C. D., Homeida, M. M., Hopkins, A. D., \& Lawrence, J. C. (2012). Elimination of onchocerciasis from Africa: possible?. Trends in parasitology, 28(1), 16-22.

Meade, M. S. (2010). Medical geography. John Wiley \& Sons, Ltd.

Meade, S. M., and Emch, M. (2010). Medical Geography. Guilford Press: New York, NY, USA; pp. 26-44.

Meade, S.M., Earickson, R.J. (2000). Medical Geography. $2^{\text {nd }}$ Edition. The Guilford Press, New York, NY, USA.

Mngulwi, B. S. (2003). Country review: United Republic of Tanzania. Review of the state of world marine capture fisheries management: Indian Ocean, 447.

Murdoch, M. E., Asuzu, M. C., Hagan, M., Makunde, W. H., Ngoumou, P., Ogbuagu, K. F., ... \& Remme, J. (2002). Onchocerciasis: the clinical and epidemiological burden of skin disease in Africa. Annals of tropical medicine and parasitology, 96(3), 283-296.

Muro, A.I., Mziray, N.R. (1990). Decline in onchocerciasis in the eastern Usambara mountains, north eastern Tanzania, and its possible relationship to deforestation. Acta Leidensia 59(12), 141-150.

National Bureau of Statistics (2011). Tanzania in Figures 2010. United Republic of Tanzania. Ministry of Finance: Dar es Salaam.

Noma, M., Zoure, H., Tekle, A. H., Enyong, P., Nwoke, B. E., \& Remme, J. H. (2014). The geographic distribution of onchocerciasis in the 20 participating countries of the African Programme for Onchocerciasis Control:(1) priority areas for ivermectin treatment. Parasit Vectors, 7(1), 325.

Okwa, O. O., Olusola, O. O. A., \& Adelani, O. F. (2009). Onchocerciasis among women in a rural Guinea savannah ecotype of Nigeria: social implications for control. Tropical Medicine and Health, 37(4), 135-140.

Pickett, K. E., \& Pearl, M. (2001). Multilevel analyses of neighbourhood socioeconomic context and health outcomes: a critical review. Journal of epidemiology and community health, 55(2), 111-122.

Plowright, R. K., Sokolow, S. H., Gorman, M. E., Daszak, P., \& Foley, J. E. (2008). Causal inference in disease ecology: investigating ecological drivers of disease emergence. Frontiers in Ecology and the Environment, 6(8), 420-429. 
Prost, A., Hervouet, J. P., \& Thylefors, B. (1979). Degree of endemicity of onchocerciasis. Bulletin of the World Health Organization, 57(4), 655-662.

Remme, J. H. F., P. Feenstra, Lever, P.R., Medici, A.C., Morel, C.M., Noma, M., Ramaiah, K.D., Richards, F., Seketeli, A., Schmunis, G., Van Brakel, W.H., Vassall, A., (2006). Tropical diseases targeted for elimination: Chagas disease, lymphatic filariasis, onchocerciasis and leprosy. Disease Control Priorities in Developing Countries, second edition. D. T. Jamison, J. G. Breman and A. R. Measham. New York, Oxford University Press: 433-449.

Say L, Raine R. (2007). A systematic review of inequalities in the use of maternal health care in developing countries: examining the scale of the problem and the importance of context. Bulletin World Health Organization, 85, 812-819.

Smith, K. F., Sax, D. F., Gaines, S. D., Guernier, V., \& Guégan, J. F. (2007). Globalization of human infectious disease. Ecology, 88(8), 1903-1910.

Tanzania Coast Management Partnership (TCMP) (2003). Coastal Management Report no. 2002. Tanzania State of Coast Report: The National ICM Strategy and Prospects for Poverty Reduction, TCMP.

Taylor, M. J., Hoerauf, A., Townson, S., Slatko, B. E., \& Ward, S. A. (2014). Anti-Wolbachia drug discovery and development: safe macrofilaricides for onchocerciasis and lymphatic filariasis. Parasitology, 141(01), 119-127.

Tekle, A. H., Zoure, H., Wanji, S., Leak, S., Noma, M., Remme, J. H., \& Amazigo, U. (2011). Integrated rapid mapping of onchocerciasis and loiasis in the Democratic Republic of Congo: impact on control strategies. Acta tropica, 120, S81-S90.

UNICEF (2012). Cities and Children: The Challenge of Urbanisation in Tanzania.

Vlahov, D., Galea, S., \& Freudenberg, N. (2005). The urban health "advantage". Journal of urban health, 82(1), 1-4.

Weldegebreal, F., Medhin, G., Weldegebriel, Z., \& Legesse, M. (2014). Assessment of community's knowledge, attitude and practice about onchocerciasis and community directed treatment with Ivermectin in Quara District, north western Ethiopia. Parasites \& vectors, 7(1), 98.

Winnen, M., A. P. Plaisier, et al. (2002). "Can ivermectin mass treatments eliminate onchocerciasis in Africa?" Bull World Health Organ 80(5): 384-91.

World Bank (2003). Reaching the rural poor: a renewed strategy for rural development. The International Bank for Reconstruction and Development/ The World Bank.

World Health Organization (2010). Conceptual and Operational Framework of Onchocerciasis Elimination With Ivermectin Treatment. African Programme for Onchocerciasis Control: Ouagadougou, Burkina Faso.

Yirga, D., Woldemichael, K., Wondafrash, M., Kassahun, W., \& Deribe, K. (2008). Knowledge and Belief about Cause and Prevention of Onchocerciasis in Bebeka, Southwest Ethiopia. Ethiopian Journal Health Science, 18, 66-68.

York, K. J., Kabole, I., Mrisho, M., Berry, D. M., \& Schmidt, E. (2015). Factors Affecting Community Participation in the CDTI Program in Morogoro, Tanzania. Journal of Nursing Scholarship, 47(1), 96-104.

Zouré, H. G., Noma, M., Tekle, A. H., Amazigo, U. V., Diggle, P. J., Giorgi, E., \& Remme, J. H. (2014). The geographic distribution of onchocerciasis in the 20 participating countries of the African Programme for Onchocerciasis Control: (2) pre-control endemicity levels and estimated number infected. Parasit Vectors, 7, 326. 
